\title{
Diagnosis and management of aspiration using fiberoptic endoscopic evaluation of swallowing in a pediatric pulmonology unit
}

\author{
Fernando Rafael Aguirregomezcorta ${ }^{1}$, Borja Osona ${ }^{2}$, Jose Peña ${ }^{3}$, Jose Antonio Gil ${ }^{1}$, \\ Susanne Vetter-Laracy ${ }^{1}$, Guillem Frontera ${ }^{1}$, Joan Figuerola ${ }^{3}$, and Cati Bover-Bauza ${ }^{1}$ \\ ${ }^{1}$ Hospital Universitari Son Espases \\ ${ }^{2}$ Hospital Universitario Son Espases \\ ${ }^{3}$ Hospital Son Espases
}

November 17, 2020

\begin{abstract}
Background and Objectives: Swallowing disorders lead to chronic lung aspiration. Early detection and treatment of aspiration in children with dysphagia is important to prevent lung damage. Diagnosis of aspiration, which may be silent, requires an instrumental study such as fiberoptic endoscopic evaluation of swallowing (FEES). Despite its usefulness, it is rarely practiced by pediatric pulmonologists. This study aimed to evaluate the feasibility and utility of FEES performed in the pediatric respiratory unit of a tertiary hospital, analyze the clinical characteristics, endoscopic findings and proposed treatments, and identify the factors associated with penetration or aspiration. Methods: Medical records of 373 children with suspected aspiration who were referred to the pediatric respiratory unit for FEES were reviewed retrospectively. Clinical characteristics, FEES findings, and the proposed treatments were analyzed. Results: Aspiration was seen in $47.9 \%$ of the patients. The most common associated conditions were neurological disease and prematurity. The most frequently observed endoscopic finding was altered laryngeal sensitivity (36.5\%). Intervention was recommended in $54.2 \%$ of the patients. Complications were not seen during any of the procedures. Conclusions: The FEES procedure performed by pediatric pulmonologists is a reliable method for diagnosing aspiration in children. It can be safely executed by trained pulmonologists, and significant endoscopic signs other than aspiration can guide in the diagnosis and management recommendations.
\end{abstract}

Diagnosis and management of aspiration using fiberoptic endoscopic evaluation of swallowing in a Pediatric Pulmonology Unit

Authors :

Fernando R. Aguirregomezcorta $\mathrm{MD}^{1,2}$, Borja Osona $\mathrm{MD}^{1,2}$, Jose A. Peña-Zarza $\mathrm{PhD}^{1,2,3}$, Jose A. Gil MD ${ }^{1,2}$, Susanne Vetter-Laracy $\mathrm{PhD}^{2,4}$, Guiem Frontera $\mathrm{MD}^{5}$, Joan Figuerola $\mathrm{PhD}^{1,2}$ Catalina Bover $\mathrm{MD}^{1,2}$

\section{Affiliations:}

${ }^{1}$ Division of Pediatric Respiratory Medicine, Department of Pediatrics, University Hospital Son Espases, Palma de Mallorca, Spain.

${ }^{2}$ Health Research Institute of the Balearic Islands (IdISBa). Department of Pediatrics. Palma de Mallorca, Spain.

${ }^{3}$ Sleep Unit, Department of Pediatrics, University Hospital Son Espases, Palma de Mallorca, Spain.

${ }^{4}$ Division of Neonatology, Department of Pediatrics, University Hospital Son Espases, Palma de Mallorca, Spain. 
${ }^{5}$ Research Unit, University Hospital Son Espases, Palma de Mallorca, Spain.

\section{Corresponding author :}

Fernando Rafael Aguirregomezcorta. Secretaría de Pediatría (planta +1). Carretera de Valldemossa, 79, 07120 Palma, Illes Balears. Phone number: +34618368311aguirrefer77@gmail.com.

Grants and financial support : None

Keywords : Children, swallowing disorders, dysphagia, chronic aspiration, fiberoptic endoscopic evaluation of swallowing

Running head : Fiberoptic endoscopic evaluation of swallowing

\section{ABSTRACT}

Background and Objectives : Swallowing disorders lead to chronic lung aspiration. Early detection and treatment of aspiration in children with dysphagia is important to prevent lung damage. Diagnosis of aspiration, which may be silent, requires an instrumental study such as fiberoptic endoscopic evaluation of swallowing (FEES). Despite its usefulness, it is rarely practiced by pediatric pulmonologists. This study aimed to evaluate the feasibility and utility of FEES performed in the pediatric respiratory unit of a tertiary hospital, analyze the clinical characteristics, endoscopic findings and proposed treatments, and identify the factors associated with penetration or aspiration.

Methods : Medical records of 373 children with suspected aspiration who were referred to the pediatric respiratory unit for FEES were reviewed retrospectively. Clinical characteristics, FEES findings, and the proposed treatments were analyzed.

Results : Aspiration was seen in $47.9 \%$ of the patients. The most common associated conditions were neurological disease and prematurity. The most frequently observed endoscopic finding was altered laryngeal sensitivity (36.5\%). Intervention was recommended in $54.2 \%$ of the patients. Complications were not seen during any of the procedures.

Conclusions : The FEES procedure performed by pediatric pulmonologists is a reliable method for diagnosing aspiration in children. It can be safely executed by trained pulmonologists, and significant endoscopic signs other than aspiration can guide in the diagnosis and management recommendations.

\section{INTRODUCTION}

Chronic pulmonary aspiration due to swallowing disorders is defined as recurrent aspiration of food, fluids, or saliva during the oropharyngeal phase of swallowing. The increase in its incidence in recent years can be attributed to prolonged survival of patients with different comorbidities.

This condition affects around $1 \%$ of the children in the general population ${ }^{1}$. However, its incidence is much higher in premature infants or those with anatomical malformations, encephalopathies, neuromuscular disorders, and genetic alterations ${ }^{2-3}$. It comprises a wide spectrum of signs and symptoms, including chronic cough, recurrent pneumonia, wheezing with poor response to treatment, atelectasis, stridor, lung abscess, chronic lung damage with bronchiectasis, and respiratory failure ${ }^{4}$. The abovementioned symptoms are frequently treated at all levels of care, ranging from the primary care pediatrician to the pediatric pulmonologist and intensive care units. Diagnosing chronic aspiration in pediatric patients is challenging. There are significant implications in the event of a delay in diagnosis. Symptoms such as coughing or other signs of aspiration are not always present during swallowing. Therefore, an instrumental evaluation, such as videofluoroscopic swallow study (VFSS) or fiberoptic endoscopic evaluation of swallowing (FEES) is recommended for the diagnosis of chronic pulmonary aspiration ${ }^{5}$.

The FEES technique has been described useful and feasible in pediatric patients for more than 20 years ${ }^{6}$. Nonetheless, it is not routinely used across all centers and countries ${ }^{7}$. This is probably due to limited availability of specialists trained in this procedure. Pediatric pulmonologists have the necessary tools and 
the anatomical and pathophysiological knowledge to perform this procedure and interpret the findings. However, many children remain undiagnosed and some of them do not receive proper treatment until the detection of chronic lung damage. Moreover, there are few studies in which the correlation between various endoscopic findings and the possibility of aspiration in pediatric patients has been extensively studied.

Therefore, in this study, we aimed to analyze the clinical characteristics, endoscopic findings, and proposed treatments in patients who underwent FEES in the pediatric pneumology unit of a tertiary hospital. We also intended to identify the factors associated with penetration/aspiration.

\section{MATERIALS AND METHODS}

We conducted a retrospective review of data of children with suspected chronic pulmonary aspiration, who underwent FEES at the pediatric pneumology department of the University Hospital Son Espases, Palma de Mallorca, Spain. The hospital serves a reference population of 1,149,460 patients, with 172,058 in the pediatric age group. Our study was approved by the Ethics Committee of Research of the Balearic Islands (IB 3876/19 PI).

We included patients aged 0-18 years, who underwent FEES between January 2006 and December 2018. In patients who underwent multiple studies, the data of the first evaluation was considered. We obtained the data using fiberoptic bronchoscope (FB), FEES database, and the hospital database that includes the clinical, laboratory, and radiology data of the patients.

The patient characteristics included were: age, sex, comorbidities, and reason for referral. The FEES procedure was performed according to the technique described by Langmore et $\mathrm{al}^{8}$. A pediatric pulmonologist conducted the procedure with the help of a pediatric nurse in the Endoscopy suite or the Pediatric/Neonatology Intensive Care Units, without displacing the critical patients. A 3-hour fasting period was maintained before the procedure. The procedure was explained to the parents and their informed consent was obtained. Our study adhered to the guiding principles of the World Medical Association Declaration of Helsinki. A flexible pediatric FB (frequently used in other situations), such as the Olympus BF-XP190@ (3.1 mm outer diameter) or the Olympus BF-N20® (2.2 mm outer diameter) was used. A pulse oximeter (Masimoß), Neuchatel, Switzerland) was placed on the patient's foot to monitor the heart rate and oxygen saturation. In addition, a drop of lidocaine/prilocaine was applied to the anterior nasal passage using a cotton swab, thereby avoiding anesthesia of the posterior area. The introduction of FB facilitated assessment of the following features: the structural and functional anatomy of the hypopharynx and larynx, pooling of secretions, and the sensitivity of larynx through air pulses or a brief contact of the endoscope with the arytenoids. This procedure was followed by a direct evaluation of swallowing. We added a drop of dye to the food for easy viewing. Different textures are used (liquid, nectar, pudding, cookie) depending on the patient's age and previous tolerance. Swallowing was observed and any abnormalities and residues were recorded. On detecting laryngeal penetration or aspiration, the pulmonologist performed different compensatory strategies, such as changes in positioning or feeding rhythm, modifications in the utensils (including bottle/nipple systems), or modifications in the viscosity of the liquids (thickening of the liquids), with the FB retained in its position. This enabled a direct assessment of the response to treatment. The tests were videotaped and subsequently evaluated with the use of slow motion.

The following variables were collected in a standardized report: anatomic abnormalities, altered laryngeal sensitivity, pharyngeal pooling of secretions, laryngeal penetration (appearance of secretions or food in the laryngeal vestibule above the vocal cords), aspiration (passage of secretions or food below the vocal cords), residue of the bolus in the hypopharynx after swallowing, proposed treatment, and its effectiveness. The post-FEES recommendations were recorded and classified into the following categories: a combined treatment comprising a change of positioning or feeding rhythms; addition of thickeners; use of omeprazole or other proton pump inhibitors (PPIs); and non-oral feeding.

Data are expressed as absolute and relative frequencies for the categorical variables and median and interquartile range for the non-normally distributed continuous variables. We conducted the Chi-square analysis or Fisher's test to determine the association of penetration/aspiration with the categorical variables. We 
used the Student t test or Mann-Whitney test for the quantitative variables.

Considering their inclusion when the probability of alpha error was $<0.1$ or when it created a confusing effect on others, we conducted a multivariate analysis on an exploratory basis by building a model of logistic regression. This helped us to assess the association of the clinical and demographic variables associated with aspiration. We determined the goodness of fit using the Hosmer-Lemshow test. The IBM SPSS (v22, NY, USA) was used for the statistical calculations.

\section{RESULTS}

A total of 472 FEES procedures were performed on 373 children with suspected aspiration. The initial FEES done for each patient was considered for the analysis.. The median age was 11 months (IQR, 3-39 months) and $58.4 \%$ of the patients were boys. Neurological disorders (34.3\%) and prematurity $(28.3 \%)$ were the most frequent underlying pathologies. A significant number of patients (19\%) had no apparent predisposing factors for aspiration. Table 1 summarizes the demographic data and the comorbidities.

The most frequent indications for the procedure were signs or symptoms of aspiration, such as choking, gagging, or coughing while feeding (46.6\%), followed by refusal or an aversion to ingest (26\%) (Table 2).

We observed abnormal swallowing resulting in penetration/aspiration in $47.9 \%$ of the cases. This rate was similar in both children born at term and those born premature (46.6\% and $51.4 \%)$. Of the 71 patients without any predisposing factors, $22.5 \%$ presented with penetration and/or aspiration.

Altered laryngeal sensitivity (36.5\%) was the most frequently observed endoscopic finding, besides penetration. Table 3 outlines the predominantly observed abnormal findings. Therapeutic action was recommended in $54.2 \%$ cases following FEES. Treatment with thickeners $(25.5 \%)$ was the most frequent recommendation. Oral feeding was contraindicated in $12.3 \%$ of the cases, thus recommending the use of a nasogastric or gastrostomy tube (Table 4). There were no significant adverse events associated with the procedures.

The multivariate logistic regression model revealed an independent association between aspiration and the predisposing factor, with alterations in laryngeal sensitivity (odds ratio [OR], 5.68; 95\% confidence interval [CI], 2.74-11.77), pharyngeal pooling (OR 11.2, 95\% CI, 3.14-40.15), and post-deglutition food residues (OR 7.89, 95\% CI 4.02-15.49). Neurological conditions, including cerebral palsy, mental delay, and encephalopathy were most significantly associated with aspiration (OR 1.99; 95\% CI 1.01-3.92), compared to those with no relevant previous pathology. Table 5 summarizes the remaining risk factors associated with aspiration.

\section{DISCUSSION}

This study examined the patient characteristics, endoscopic findings, and proposed treatments in children with suspected aspiration, evaluated by FEES in a pediatric pneumology unit.

We observed penetration or aspiration in nearly half of the cases. Our data were similar to those obtained in other pediatric studies ${ }^{9}$. Nonetheless, their incidence varies according to the pathology and age group, and was found to be greater in patients with neurological disorders ${ }^{10}$.

Our study included pediatric patients of all age groups and it needs to be highlighted that a large proportion of these were premature children. (105 of 373 patients). This reinforces the value of FEES in this specific population with high rates of pulmonary aspiration ${ }^{11}$, and its application in breastfeeding infants ${ }^{12}$.

FEES does not affect regular swallowing. Pharyngeal contraction causes white-out and completely might block visualization of all oropharyngeal structures for a brief period. As a result, food bolus movement is not directly observed. A close inspection of the glottis and sub glottis may reveal the aspirated material. If not directly observed, other endoscopic signs provide information about a possible impairment of normal swallowing.

Pharyngeal pooling of secretions, which is always an abnormal finding correlated the most with aspiration, with an OR of 11.2. Saliva aspiration is probably the least frequently reported form of chronic aspiration. Saliva contains potentially pathogenic oral microorganisms which can alter the lung microbiome, if the 
amount aspiration is significantly higher ${ }^{13}$. Moreover, it can cause pneumonia ${ }^{14}$. Salivary aspiration observed during FEES has also been associated with other radiological alterations, such as bronchial thickening and atelectasis ${ }^{15}$. Salivary aspiration in children with neurological disorders is generally caused by a severe incoordination of swallowing and reduced sensitivity of the laryngopharynx, rather than due to excessive production of saliva.

Correspondingly, our study revealed a substantial association between impaired laryngeal sensitivity in children and aspiration $(\mathrm{OR}=5.6)$. This can be associated with the frequent alteration of sensitivity in children with dysphagia ${ }^{16}$, which could be attributed to gastro-esophageal reflux ${ }^{17}$.

The presence of post-swallow residue in the vallecula and/or piriform sinuses is an endoscopic sign indicating an abnormal clearance of the alimentary bolus. Therefore, we identified its correlation with aspiration, consistent with other studies ${ }^{18}$. This might be the result of an altered pharyngeal constriction, tongue retraction, or laryngeal displacement ${ }^{19}$.

Despite the significance of a good clinical evaluation prior to endoscopic examination, its low sensitivity for aspiration detection ${ }^{20}$ makes it relatively unhelpful for the pediatric pulmonologist. Furthermore, the great frequency of silent aspiration in pediatrics ${ }^{21}$ calls for the need of a complementary instrumental study. VFSS and the FEES ${ }^{22}$ are the two most recommended complementary studies. Several studies have compared the diagnostic benefits of the aforementioned techniques. Notwithstanding their similar specificities, FEES is slightly more sensitive for detecting penetration, aspiration, and residues, compared to VFSS ${ }^{23-24}$.

Despite the complementary nature of these techniques, FEES has the following advantages: real food and drinks are used, there is no restriction on the duration of the test; it can be repeated several times during follow-up, parents can observe the study, and the equipment used for the procedure is portable, allowing FEES to be performed in intensive care units as well.

However, the absence of radiation is its primary advantage, particularly in children and premature patients. The radiation dose in VFSS is relatively low, between 0.2 and $0.85 \mathrm{mSv}^{25}$. A chest X-ray usually involves a receiving radiation of $0.02 \mathrm{mSv}$. Nonetheless, the need to repeat studies during follow-up increases this radiation to the equivalent of more than 30 chest X-rays ${ }^{26}$. Seventy-four patients (19.8\%) required follow-up with repeated FEES in our study.

The multidisciplinary approach to dysphagia implies enormous variability in the diagnostic methods used in the USA, Australia, and Ireland ${ }^{27-29}$. Despite the highlighted advantages of FEES, VFSS is reportedly the most widely used diagnostic method. Approximately 36-40\% of clinical assessments are followed by VFSS in Canada and the USA, with a slight difference in Australia and Japan. Other instrumental assessments, such as FEES are not used as frequently as $\mathrm{VFSS}^{30}$. This can be due to different reasons associated with endoscopy, such as the non-availability of equipments, lack of knowledge of the technique, lack of specialists, or different legislations that regulate handling of the endoscope.

FEES is usually performed by otolaryngologists or speech pathologists in the US, Japan, Canada, and some European countries ${ }^{19}$. However, it is also performed by neurologists in other countries such as Germany ${ }^{31}$. The ideal assessment of dysphagia should be done by a multidisciplinary team. Pediatric pulmonologists are skilled in flexible endoscopy and in the identification of structural and functional abnormalities of the airway. This provides an optimal setting for FEES examinations. Furthermore, they have the advantage of being one of the most qualified specialists to relate the findings to the underlying medical problem. To this end, it would be desirable to carry out standardized and specific complementary training in this area open to all clinicians, as also proposed by other authors ${ }^{31}$. Adequate training ensures excellent safety of the technique ${ }^{32}$.

We also found aspiration during FEES in a subgroup of patients, without any predisposing factor or underlying pathology. The abovementioned findings have been previously reported ${ }^{33}$. Nonetheless, the possibility of aspiration in this population is underestimated.

Our study has several limitations. First, the retrospective and single center design made it susceptible to selection bias. Second, the patient sample was heterogeneous. Therefore, it might create difficulty while 
extrapolating our results to concrete patient populations. Nevertheless, the variability of the pathologies and ages represents the kind of patients commonly encountered in a pediatric hospital. Third, we attempted to adjust for the important medical conditions that increase the risk of aspiration. However, we may have not accounted for other confounding factors. For example, despite similar neurologic comorbidity labels in several patients, they might have extremely different functional realities.

In conclusion, performing FEES in a pediatric pneumology unit is useful and safe. Almost half of our patients who underwent FEES suffered from penetration/aspiration. Direct or indirect identification of aspiration is within the reach of pediatric pulmonologists with prior training. This in turn will enable early diagnosis and avoid advanced lung damage. In addition, it will provide information on the efficacy of compensatory swallowing strategies and guide treatments.

Conflicts of interest : The authors declare no conflict of interest.

\section{REFERENCES}

1. Bhattacharyya N. The prevalence of pediatric voice and swallowing problems in the United States. Laryngoscope 2015; 125:746-750

2. Jadcherla S. Dysphagia in the high-risk infant: potential factors and mechanisms. Am J Clin Nutr . 2016;103(2):622S-8S.

3. Bae SO, Lee GP, Seo HG, Oh BM, Han TR. Clinical characteristics associated with aspiration or penetration in children with swallowing problem. Ann Rehabil Med . 2014;38(6):734-741

4. Tutor, J.D. and Gosa, M.M. Dysphagia and aspiration in children. Pediatr. Pulmonol. 2012; 47: 321-337

5. Boesch R.P, Daines C, Willging J.,P Kaul A, Cohen A,P,Wood R.E, Amin R.S.. Advances in the diagnosis and management of chronic pulmonary aspiration in children. Eur Respir J. 2006; 28:847-61

6. Hartnick CJ, Hartley BE, Miller C, Willging JP. Pediatric fiberoptic endoscopic evaluation of swallowing. Ann Otol Rhino Laryngol.2000;109:996-999.

7. C Wallis, M Ryan. Assessing the Role of Aspiration in Pediatric Lung Disease. Pediatr Allergy, Immunol Pulmonol. 2012;132-142

8. Langmore SE, Schatz K, Olsen N. Fiberoptic endoscopic evaluation of swallowing safety: a new procedure. Dysphagia 1988; 2:216-219

9. Leder, SB, Karas, DE. Fiberoptic endoscopic evaluation of swallowing in the pediatric population. Laryngoscope. 2000; 110:1132-1136

10. Beer S, Hartlieb T, Müller A.D, Granel M, Staudt M. Aspiration in children and adolescents with neurogenic dysphagia: comparison of clinical judgment and fiberoptic endoscopic evaluation of swallowing. Neuropediatrics 2014; 45: 402-5

11. Suterwala, M., Reynolds, J., Carroll, S. ,Sturdivant C,ArmstrongES. Using fiberoptic endoscopic evaluation of swallowing to detect laryngeal penetration and aspiration in infants in the neonatal intensive care unit. J Perinatol 2017;37: 404-408

12. Willette, S., Molinaro, L.H., Thompson, D.M. and Schroeder, J.W., Jr. Fiberoptic examination of swallowing in the breastfeeding infant. Laryngoscope. 2016; 126: 1681-1686

13. Duvallet C, Larson K, Snapper S, Iosim S, Lee A, Freer K, May K, Alm E, Rosen R. Aerodigestive sampling reveals altered microbial exchange between lung, oropharyngeal, and gastric microbiomes in children with impaired swallow function. PLoS One. 2019;14: e0216453

14. Link DT, Willging JP, Miller CK, Cotton RT, Rudolph CD. Pediatric laryngopharyngeal sensory testing during flexible endoscopic evaluation of swallowing: feasible and correlative. Ann Otol Rhinol Laryngol. 2000; 109:899-905

15. Tanaka N., Nohara K., Ueda A., Katayama T, Ushio M., Fujii N, Sakai T.Effect of aspiration on the lungs in children: a comparison using chest computed tomography findings. BMC Pediatr 2019;19: 162

16. Ulualp S, Brown, A, Sanghavi R, Rivera-Sanchez Y. Assessment of laryngopharyngeal sensation in children with dysphagia. Laryngoscope; 2013: 123: 2291-2295

17. Aviv JE, Liu H, Parides ST, Close LG. Laryngopharyngeal sensory deficits in patients with laryngopharygeal reflux and dysphagia. Ann Otol Rhin Laryn 2000; 109:1000-1006 
18. Neubauer, P.D., Hersey, D.P., Leder, S.B. Pharyngeal Residue Severity Rating Scales Based on Fiberoptic Endoscopic Evaluation of Swallowing: A Systematic Review. Dysphagia 2016;31: 352-359

19. Langmore, S.E. History of Fiberoptic Endoscopic Evaluation of Swallowing for Evaluation and Management of Pharyngeal Dysphagia: Changes over the Years. Dysphagia ; 2017:32, 27-38

20. Duncan DR, Mitchell PD, Larson K, Rosen RL. Presenting Signs and Symptoms do not Predict Aspiration Risk in Children. J Pediatr . 2018; 201:141-146

21. Weir K, McMahon S, Taylor S, Chang A. Oropharyngeal Aspiration and Silent Aspiration in Children. Chest. 2011; 140:589-597

22. Espitaliera, F., Fanousb, A., Avivc, J., Bassiounyd, S., Desutere, G., Nerurkarf, N., et al. International consensus International consensus (ICON) on assessment of oropharyngeal dysphagia. Eur Ann Otorhinolaryngol Head Neck Dis. 2018;135: S17-S21

23. Giraldo-Cadavid LF, Leal-Leano LR, Leon-Basantes GA, Bastidas AR, Garcia R, Ovalle S, AbondanoGaravito J E.. Accuracy of endoscopic and videofluoroscopic evaluations of swallowing for oropharyngeal dysphagia. Laryngoscope 2017; 127:2002-10

24. da Silva AP, Lubianca Neto JF, Santoro PP. Comparison between videofluoroscopy and endoscopic evaluation of swallowing for the diagnosis of dysphagia in children. Otolaryngol Head Neck Surg. 2010; 143:204-209

25. Lo Re G, Vernuccio F, di Vittorio ML, Scopelliti L, Di Piazza A, Terranova M.C, Picone D, Tudisca C, Salerno S.. Swallowing evaluation with videofluoroscopy in the paediatric populationSwallowing evaluation with videofluoroscopy in the paediatric populationSwallowing evaluation with videofluoroscopy in the paediatric populationSwallowing evaluation with videofluoroscopy in the paediatric population. Acta Otorhinolaryngol Ital. 2019; 39: 279-288

26. Hersh C, Wentland C, Sally S, de Stadler M, Hardy S, Fracchia MS, Hartnick C. Radiation exposure from videofluoroscopic swallow studies in children with a type 1 latyngeal cleft and pharyngeal dysphagia: a retrospective review. Int J Pediatr Otorhinolaryngol. 2016; 89:92-96

27. Mathers-Schmidt, B.A., Kurlinski, M. Dysphagia Evaluation Practices: Inconsistencies in Clinical Assessment and Instrumental Examination Decision-Making. Dysphagia; 2003:18, 114-125

28. Howells, S.R., Cornwell, P.L., Ward, E.C., Kuipers P. Understanding Dysphagia Care in the Community Setting. Dysphagia. 2019; 34: 681-691

29. Pettigrew, C.M., O'Toole, C. Dysphagia Evaluation Practices of Speech and Language Therapists in Ireland: Clinical Assessment and Instrumental Examination Decision-Making. Dysphagia .2007;22: 235244

30. Gonzalez-Fernandez M, Huckabee ML, Doeltgen SH, Inamoto Y, Kagaya H, Saitoh E. Dysphagia Rehabilitation: Similarities and Differences in Three Areas of the World. Curr Phys Med Rehabil Rep . 2013; 1:296-306

31. Dziewas R, Glahn J, Helfer C, Ickenstein G, Keller J, Ledl C, Lindner-Pfleghar B, Nabavi D G, Prosiegel M, Riecker A et al.. Flexible endoscopic evaluation of swallowing (FEES) for neurogenic dysphagia: training curriculum of the German Society of Neurology and the German stroke society. BMC Med Educ. 2016; 16:70

32. Miller CK, Willging JP. Fiberoptic Endoscopic Evaluation of Swallowing in Infants and Children: Protocol, Safety, and Clinical Efficacy: 25 Years of Experience. Ann Otol Rhinol Laryngol. 2020; 129:469-481

33. Lefton-Greif, M.A., Carroll, J.L, Loughlin, G.M. Long-term follow-up of oropharyngeal dysphagia in children without apparent risk factors. Pediatr. Pulmonol. 2006; 41: 1040-1048.

\section{Hosted file}

tables12.pdf available at https://authorea.com/users/376386/articles/493352-diagnosis-andmanagement-of-aspiration-using-fiberoptic-endoscopic-evaluation-of-swallowing-in-apediatric-pulmonology-unit 